\title{
Design of a Electrically Tunable Filter for RF Front-end Circuitry of the Microwave Receiver
}

\author{
WU Qing-ping \\ Changzhou College of information Technology \\ Changzhou, China \\ E-mail: czwqp@sina.com
}

\author{
SHEN Kai \\ Changzhou College of information Technology \\ Changzhou, China \\ E-mail: shelly_sk@sina.com
}

\author{
ZHANG Hui-min \\ Changzhou College of information Technology \\ Changzhou, China \\ E-mail: 13138955@qq.com \\ ZHOU Yu-feng \\ Changzhou Radio Factory Limited Company \\ Changzhou, China \\ E-mail: czrfzyf1964@sina.com
}

\begin{abstract}
The electrically tunable filter for the RF front-end circuitry of the microwave receiver is designed to the performance with low insertion loss, low volatility band, high signal selection and superior adjacent channel interference. This paper presents a design scheme of a 225 to $500 \mathrm{MHz}$ electrically tunable filter. The design uses the capacitive coupled double tuned resonant circuit than can make the signal has a higher selectivity at the resonant frequency and the varactor-BB178 is chosen in order to achieve tuning characteristics of the electrical filter. Based on the principle of power transfer filter and variable capacitance diode characteristics, a theoretical analysis was made for the influence to the important indexes of the frequency Variation to the electric adjustable filter insertion loss and rectangle coefficient. The circuit design is optimized simulation by Professional simulation software ADS and the filter waveform at different resonant frequencies was received. Designed by the program, the filter's $3 \mathrm{~dB}$ bandwidth is greater than $10 \mathrm{MHz}$, stop-band attenuation is greater than $30 \mathrm{~dB}$, insertion loss is less than $7 \mathrm{~dB}$, standing wave ratio (VSWR) is less than 1.5, can effectively improve the signal to noise ratio of the receiver, and can solve the problem of interference and electromagnetic compatibility when multiple radio equipment work at the same time.
\end{abstract}

Keywords- microwave, electrically tunable filter, variable capacitance diode, quality factor, insertion loss

\section{INTRODUCTION}

With the electromagnetic environment becoming increasingly complex, anti-interference requirements for radio communications is becoming increasingly high. For example, when multiple microwave receivers operate simultaneously, due to the short distance, they will interfere with each other. Severe, can lead to the channel can not be used due to obstruction [1]. Currently, most of the receivers using fixed bandwidth band pass filter bank groups in the front-end RF circuit to improve anti-jamming capability [2], But When a wide frequency band received, this design will lead to surge in the number of filters that is not conducive to cost control and compact design of the receiver. To meet the actual needs, the filter must be low insertion loss, low volatility band, high signal selectivity, while the volume as small as possible, in order to meet the requirements of sensitivity and dynamic range. The electrically tunable filter has advantages of small volume and wide frequency band that the second order composite signal can be well suppressed to improve the selectivity and anti-jamming capability of the receiver [3]. The core device of electrically tunable filter is the variable capacitance diode. If both ends of the variable capacitance diode reverse bias are changed, the capacitance value of the variable capacitance diode will change too. So the center frequency of the filter will change, the change in frequency can be quickly tracked [4]. This paper presents a design scheme of a 225 to $500 \mathrm{MHz}$ electrically tunable filter. This electrically tunable filter can effectively improve the SNR of the receiver, and can solve the interference and EMC problems caused by multiple microwave receivers operating simultaneously.

\section{DESIGN OF THE ELECTRICALLY TUNABLE FILTER CIRCUIT}

In order to have better selectivity, usually the dual tuning circuit is used in the electrically tunable filter. There two forms with series resonance and parallel resonance in the tuned circuit, respectively coupled by inductance or capacitance [5]. Since the series resonant bias circuit is complex, and the stray capacitance of the inductor can not easily be absorbed, so the design uses a parallel resonant circuit with capacitive coupling.

\section{A. Amplitude-frequency characteristics analysis of the capacitively coupled double tuned circuit [6]}

Fig .1 is a equivalent circuit diagram of the electrically tunable filter through capacitive coupling. Wherein, $C_{\mathrm{M}}$ is the coupling capacitor, $L_{1} 、 C_{1}$ and $L_{2} 、 C_{2}$ constitute the 
dual resonant circuit together. In the practical electrically tunable filter circuit, $C_{1}$ and $C_{2}$ are usually replaced by the variable capacitance diode.

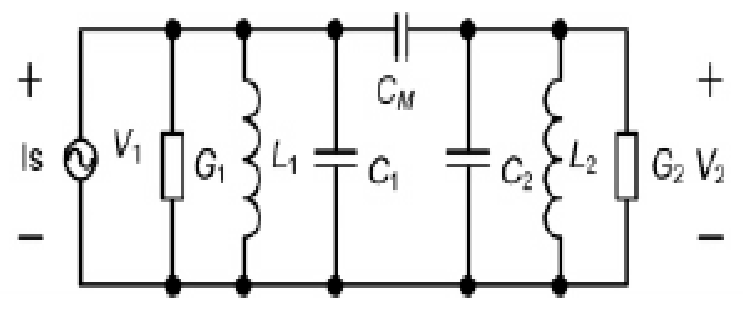

Figure 1. The circuit consists of capacitive coupling double-tuned circuit

To facilitate debugging, we usually choose $L_{1}=L_{2}=L$, $C_{1}=C_{2}=C, G_{1}=\mathrm{G}_{2}=\mathrm{G}$, and satisfies $C \gg C_{\mathrm{M}}$, In this case, the coupling coefficient tuning loop $k \approx C_{\mathrm{M}} / C$.

By circuit node voltage method, two nodes voltage equation are listed in Fig .1:

$$
\begin{aligned}
& I_{s}=\left(G+j \omega C+j \omega C_{M}+\frac{1}{j \omega L}\right) V_{1}-j \omega C_{M} V_{2} \\
& 0=\left(G+j \omega C+j \omega C_{M}+\frac{1}{j \omega L}\right) V_{2}-j \omega C_{M} V_{1}
\end{aligned}
$$

The amplitude-frequency characteristics of the tuning circuit can be obtained:

$$
\frac{\mathrm{V}_{2}}{\mathrm{I}_{\mathrm{s}}}=\frac{4 \eta \mathrm{G}}{\sqrt{\left(1-\varepsilon^{2}+\eta^{2}\right)^{2}+4 \varepsilon^{2}}}
$$

Among them : $\varepsilon=\mathrm{Q}\left(\frac{\mathrm{f}}{\mathrm{f}_{0}}-\frac{\mathrm{f}_{0}}{\mathrm{f}}\right)$ is a generalized detuning, $\mathrm{Q}$ is the quality factor of the circuit, $f_{0}$ is the resonance frequency. $\eta=\mathrm{Qk}=\frac{\omega \mathrm{C}_{\mathrm{M}}}{\mathrm{G}}$ is the coupling factor. When $\eta<1$, the circuit is a weak coupling state; if $\eta=1$, the circuit is a critical coupling state; when $\eta>1$, the circuit is a strongly coupled state.

If the value of the coupling capacitance $C_{\mathrm{M}}$ fixed, the coupling factor will change as the center frequency, the dual-tuned circuit will produce a change process from weak coupling to strong coupling [7]. And the larger the coupling factor is, the wider the tuning bandwidth is, but also the greater the fluctuation band increases. Thus, when the center frequency is adjusted, the gain and the $3 \mathrm{~dB}$ bandwidth will change, so the electrically tunable filter is only suitable for smaller tuning range of occasions.

\section{B. Design of the electrically tunable filter with the adjustable coupling capacitor}

In order to ensure the coupling factor to maintain a relatively constant value when the center frequency is changed, the coupling capacitance is replaced by the variable capacitance diode. Fig .2 is a circuit configuration of the electrically tunable filter.

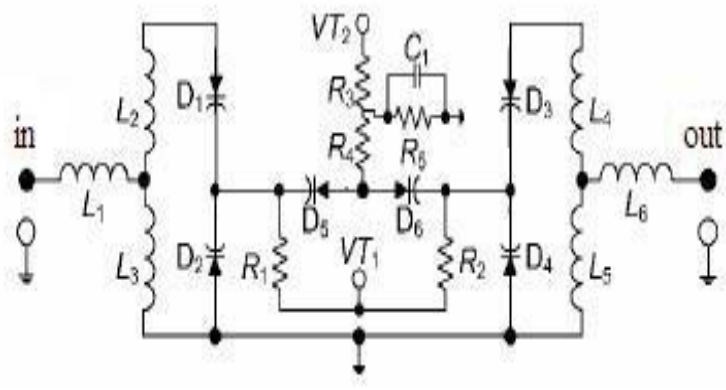

Figure 2. the schematic of the electrically tunable filter with the adjustable coupling capacitor

The capacity-coupled double-tuned circuit is used in the electrically tunable filter based on the high selectivity of the receiver requirements. Because the variable capacitance diode may be used as the tuning element in the tuning circuit, we can adjust the reverse bias voltage of the variable capacitance diode to change the capacitance value of the resonant circuit so as to adjust the center frequency of the filter. In the actual circuit, the variable capacitance diode is usually connected in the form of back to back tubes that can eliminate the fundamental frequency harmonics generated by the variable capacitance diode to effectively reduce crosstalk [8]. Tuning variable capacitance diode D1, D2, D3, D4 are provided reverse bias voltage by VT1. The reverse bias voltage across coupling variable capacitance diode D5, D6 can be obtained by VT1, VT2 subtraction.

To select variable capacitance diode must focus on several parameters below [9]: First, its capacitance variation ratio must be large, at the same voltage change, the more the capacitance variation ratio is, the greater the adjustable frequency range of the filter is; Second, its $\mathrm{Q}$ value muse be higher, this corresponds to the series resistance of the variable capacitance diode must be smaller, because the series resistance of the variable capacitance diode has a very significant impact on the insertion loss of the filter; thirdly, the Series inductance must be as small as possible, because the series inductance can cause an offset of the center frequency to the filter. In addition, the self-resonant frequency of the variable capacitance diode must be higher than the selectable frequency range of the filter to ensure that the filter can work well. In selecting variable capacitance diode time, another point is often overlooked, which is the reverse cut-off current of the variable capacitance diode. When the variable capacitance diode usually being reverse biased, the bias circuits is generally connected divider resistance or protective resistance. If the resistance value is relatively large, the entire circuit will be given additionally not small power consumption. This is 
detrimental to the circuit design, so the reverse cut-off current of the variable capacitance diode should be selected smaller. For these reasons, the variable capacitance diode used in the design is Philips BB178. The relationship of its capacitance changes with reverse voltage is shown in Fig .3.

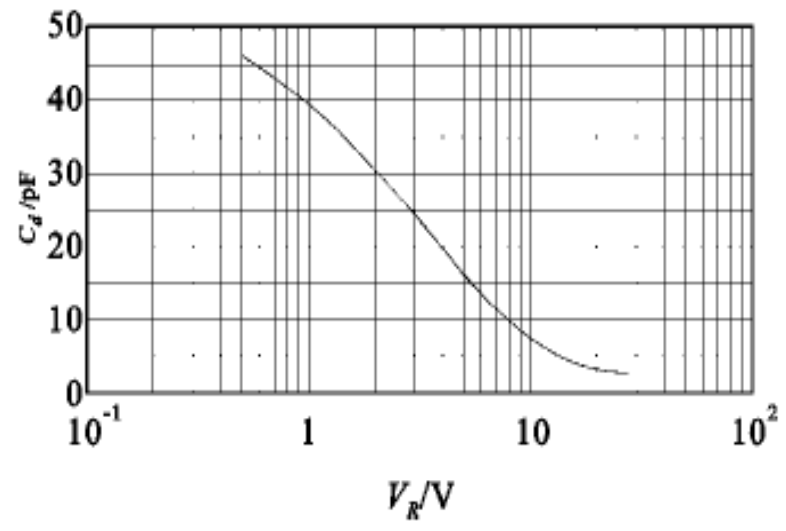

Figure 3. the BB178 capacitance value's relationship with the reverse voltage

As can be seen from Fig .3, the range of capacitance values is $2.7 \mathrm{pF} \sim 46 \mathrm{pF}$. The frequency range of the tunable filter is up to:

$$
\sqrt{\mathrm{C}_{\max } / \mathrm{C}_{\min }}=\sqrt{46 / 2.7}=4.12
$$

And the filter frequency range designed

$$
\mathrm{f}_{\max } / \mathrm{f}_{\min }=500 / 225=2.22
$$

Obviously,

$$
\sqrt{\mathrm{C}_{\max } / \mathrm{C}_{\min }} \geq \mathrm{f}_{\max } / \mathrm{f}_{\text {min }}
$$

It satisfies the design requirements.

In order to improve the $\mathrm{Q}$ value of the resonant circuit, inductance taps section access is used in the design of the resonant circuit in order to prevent the impact of input and output load on the $\mathrm{Q}$ value of the resonance circuit. At the same time, the higher $\mathrm{Q}$ value inductors must be chosen. Because $\mathrm{Q}$ values of the general color ring inductors and chip inductor are low, so the coil inductance wound by the enameled wire is chosen for the resonant inductor in the design. Thus, we can easily adjust the indicators of the resonant circuit by adjusting the density of the coil.

\section{SIMULATION AND TEST FOR THE ELECTRICALLY TUNABLE FILTER}

S-parameter is a set of linear relations established by the incident and reflected wave that is typically used in microwave circuits analyze and describe the input characteristics of the network. In this paper, the ADS (Advanced Design System) simulation software developed by Agilent is used to simulate the electrically tunable filter circuit shown in Fig .2 for S-parameter. The tuning tool of ADS allows the user to change one or more design parameters of the filter circuit, without re-simulation its effect can be immediately observed on the output of the circuit or system [10]. Not only that, the performance of the filter can also be evaluated through simulation.

Before the simulation, the simulation models and design parameters need to be established. In this article, the model for the electrically tunable filter circuit is established to be simulated by ADS. The S21 simulation result with its center frequency between $225 \mathrm{MHz}$ and $500 \mathrm{MHz}$ is shown in Fig .4 and Fig .5.

The electrically tunable filter is test by the E5070B network analyzer. Test results show that at the tuning frequency range of $225 \mathrm{MHz} \sim 500 \mathrm{MHz}, 3 \mathrm{~dB}$ bandwidth was greater than $10 \mathrm{MHz}$, the stop attenuation $\left(\mathrm{f}_{0} \pm 50 \mathrm{MHz}\right)$ was greater than $30 \mathrm{~dB}$, insertion loss was less than $7 \mathrm{~dB}$, standing-wave ratio (VSWR) was less than 1.5 , the performance required by electrically tunable filter is achieved.

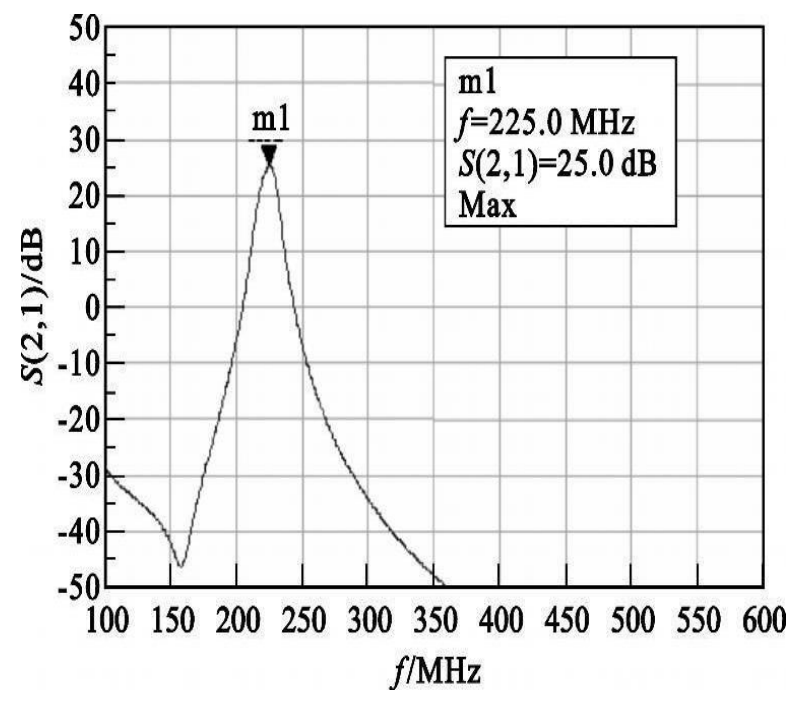

Figure 4. S-parameter simulation results of the filter with its center frequency at $225 \mathrm{MHz}$

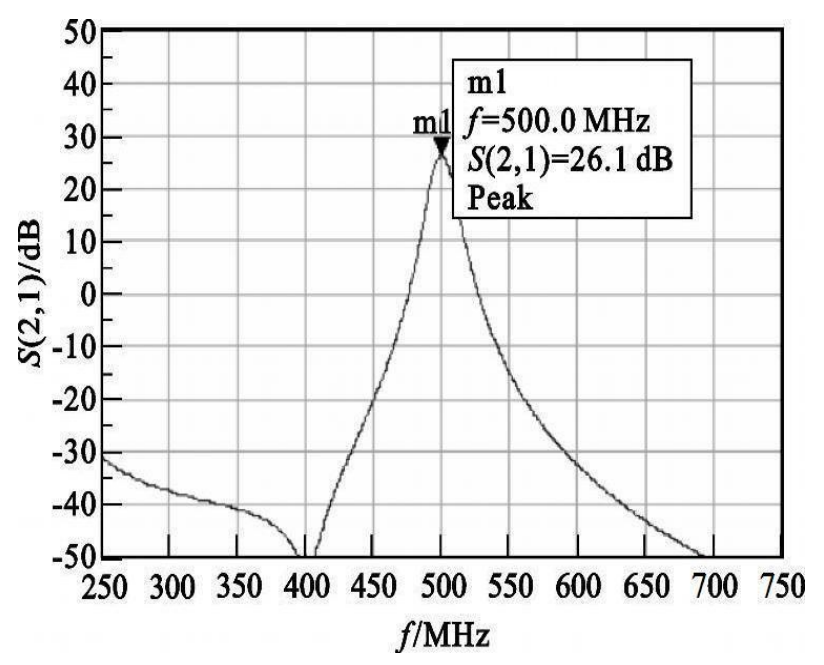

Figure 5. S-parameter simulation results of the filter with its center frequency at $500 \mathrm{MHz}$ 
However, insertion loss of the test was somewhat less than the simulation results. On the one hand it is due to the impact of parasitic resistors of the inductance, on the other hand it is that input and output ports of the filter are not exactly match that affect the test result in the cable and connectors when debugging. Further, the rectangular coefficient of the test was also slightly lower than the simulation result, which is due to the $Q$ value of the variable capacitance diode and the inductance was lower. As for that the insertion loss is larger than the design requirements is due to that dielectric loss of the double-sided CCL was large and $Q$ value of the variable capacitance diode was small.

\section{CONCLUSION}

The electrically tunable filter is an important and indispensable device of the electronic warfare equipment, the electrically tuned filter designed in this paper has low power consumption, good selectivity and good consistency of different electrical parameters of the frequency characteristics. That series resistance and inductance of the variable capacitance diode have a greater impact on the simulation results was found in the design. So if we choose a smaller series resistance and high $Q$ value variable capacitance diode, some impact will be caused on performance of the filter, particularly reflected in the return loss parameters. Further, the influence of the bias circuit can be reduced to the filter bandwidth by improving the equivalent series resistance of the bias circuit. This filter is small with simple debugging, low cost and high availability. Its main performance all meets the design requirements. It has been successfully applied in a microwave receiver and has good application prospects.

\section{ACKNOWLEDGMENT}

This work is supported by the industrial scientific and technological project in Changzhou City, Jiangsu Province, China (CE20130035).

\section{REFERENCES}

[1] Prophet E.M, Musolf J, Zuck B.F, Jimenez S, Kihlstrom, K.E and Willemsen, B.A. Highly-selective electronically-tunable cryogenic filters using monolithic discretely-switchable MEMS capacitor arrays[J] , IEEE Transactions on Applied Superconductivity,2010, 15(2): 956-959.

[2] Lin Xiaohui. Research on improving the spur-free dynamic range of receiver [J]. Radio Communications Technology, 2009,35( 3) : 4951

[3] Shi Yong hot, Chen Ji months. CNC hopping electric tunable filter design [J]. Electro-acoustic technology, 2009,33 (9): 34-38.

[4] Shuzuo Lou and Howard C. Luong. A linearization technique for RF receiver front-end using second-order-intermodulation injection[J]. IEEE Journal of solid-state circuits, 2008, 43(11) : 2404-2412.

[5] Chen Yongtai, Liu Quan. Communication electronic circuits principle and applicaton[M]. Beijing: Higher Education Press, 2011.

[6] Cheng zhiqun, Zhang Sheng, Li Jin, Zhou Pengfei. Broadband wireless communication RF transceiver front-end design [J] electronic devices .2010 (2): 186-188.

[7] PENG Zhihua, ZOU Xiaoping. An Improved Design of Electric Tuned RF Filter[J]. Radio Communications Technology, 2012(3):78-80.

[8] You Zhigang, Lin Qixian, Deng lIKE. Research and Design of electrically tunable filter [J]. Communications technology, 2011 (1): 163-167.

[9] Zhang Junmei. Development of highly selective front-end filters for the VHF receiver [D] Wuhan: Wuhan University of Technology, 2012.

[10] Feng Xinyu, Che Xiangqian, Mu Xiuchun. Design and simulation of ADS2009 RF circuit[M]. Beijing: Electronic Industry Press, 2010 